\title{
Efectos de diferentes fracciones de harinas de trigo pan obtenidas con molino industrial sobre la calidad de galletitas dulces
}

Barrera, G.N.; E. Bassi, R.J. Reyes Martínez, A.E. León y P.D. Ribotta

\begin{abstract}
RESUMEN
Se evaluaron las propiedades físico-químicas y calidad galletitera de distintas fracciones de harina de trigo pan obtenidas por molienda industrial, y se compararon con una harina de trigo pan obtenida por molienda experimental. Se determinó que los procedimientos de molienda incidieron sobre el contenido de almidón dañado y el porcentaje de cenizas en las harinas. Se observó un incremento en el contenido de almidón dañado y una disminución en el contenido de cenizas. Se registraron variaciones significativas en el perfil de capacidad de retención de solventes (SRC) e Índice de sedimentación en dodecil sulfato de sodio (IS-SDS). El factor galletita (FG), la dureza, la rugosidad superficial y la fracción de área de agrietamiento de las galletitas mostraron cambios significativos según la fracción de harina utilizada. El proceso de molienda afectó significativamente la capacidad de las harinas para la elaboración de galletitas. El análisis de componentes principales permitió discriminar entre las diferentes fracciones de harinas a partir de las evaluaciones fisicoquímicas y reológicas, lo que indica que la combinación de ambos tipos de determinaciones permite predecir el comportamiento tecnológico de una harina para la elaboración de galletitas.
\end{abstract}

Palabras clave: molienda, calidad, galletitas

Barrera, G.N.; E. Bassi, R.J. Reyes Martínez, A.E. León and P.D. Ribotta, 2012. Effects of different fractions of wheat flour produced in an industrial mill on cookies quality. Agriscientia XXIX (2): 69-79

\section{SUMMARY}

The physicochemical properties and quality of four fractions of wheat flour produced in an industrial mill and compared with wheat flour obtained by experimental milling were evaluated. The milling process modified damaged starch and ash content of the flours. An increase in the damaged starch content and a decrease in the ash content were observed. Significant variations in solvent retention capacity profile (SRC) and sodium dodecyl sulfate sedimentation index (SDS-SI) were recorded. The cookie factor (CF), hardness, surface roughness 
and the area of cracking of the cookies showed significant changes depending on the fraction of flour tested. The milling process significantly affected the ability of the flour for making cookies. The principal component analysis was used to discriminate between the different fractions of flours, from this; it is possible to conclude that the combination of physicochemical and rheological evaluations can predict the technological flour behavior to make cookies.

Key words: mill, cookies, quality

G.N. Barrera (ICYTAC, CONICET), A.E. León (ICYTAC, CONICET), P.D. Ribotta (ICYTAC, CONICET) y R.J. Reyes Martínez: Facultad de Ciencias Agropecuarias, Universidad Nacional de Córdoba, Avenida Valparaiso y Rogelio Martínez, C.C. 509, C.P. 5000, Córdoba, Argentina. E. Bassi: Molino Carlos Boero Romano S.A.I.C., San Francisco, Córdoba. Correspondencia a P. D. Ribotta: pribotta@agro.unc.edu.ar

\section{INTRODUCCIÓN}

La harina de trigo es el producto obtenido por la molienda gradual y sistemática de los granos de trigo, previa separación de las impurezas hasta un grado de extracción determinado.

La molienda es un proceso mecánico que involucra la separación del salvado y el germen del endospermo. El proceso gradual de ruptura del grano de trigo, la recuperación de endospermo adherido al salvado y, finalmente, la reducción del endospermo a harina, generan innumerables fracciones de productos en un diagrama de molienda industrial. Posteriormente a cada etapa de reducción de tamaño del grano, el material es tamizado con el propósito de separar las distintas fracciones, primero según su tamaño y, posteriormente, de acuerdo a su densidad (Hoseney, 1994; Webb \& Owens, 2003).

La separación de la harina se lleva a cabo en cernedores planos (plansifters). Cualquier material que pasa a través de los tamices más finos es, por definición, harina. El resto de material se devuelve al punto apropiado del sistema para su posterior procesamiento. Las múltiples fracciones obtenidas pueden poseer propiedades diferenciales y ser mezcladas en su totalidad para lograr una harina global o clasificadas para realizar mezclas especiales que permitan obtener las diferentes harinas. Las cantidades producidas en cada etapa varían ampliamente entre los distintos molinos. Los ajustes operacionales dependen de factores tan diversos como el tipo de trigo, el operador de la planta, las demandas del cliente, el proveedor de equipos, la ubicación geográfica e, incluso, la tradición. Existen diferencias importantes entre los productos de los molinos industriales, debido a la intensidad con que se aplican los procesos y las configuraciones utilizadas en las máquinas particulares (Webb \& Owens, 2003).

La composición y calidad de la harina producida a través del proceso de molienda depende de la secuencia de operaciones llevadas a cabo y del grado de separación logrado. Dentro del grano de trigo, los componentes mayoritarios no se encuentran distribuidos uniformemente, lo que da lugar a variaciones en la composición y funcionalidad de las distintas fracciones de harina (Dewettinck et al., 2008). El tamaño de partícula, los niveles de cenizas, el porcentaje de proteínas, la actividad enzimática y el contenido de almidón dañado, de las diferentes fracciones son afectados por el flujo de molienda (Ruffet, 1994; Prabhasankar et al., 2000).

Una clasificación posible de las harinas es en función del contenido de salvado que contienen éstas. El salvado está formado por el pericarpio, la capa de aleuronas, la epidermis nucelar y la testa (Hoseney, 1994), y dada su composición química, contiene una elevada proporción de minerales y de fibras. Por lo tanto, el contenido de cenizas permite clasificar las harinas en función del grado de extracción (kg de harina obtenidos cada $100 \mathrm{~kg}$ de trigo molido). En consecuencia, un bajo contenido de cenizas en la harina indica un bajo nivel de contaminación con salvado.

Durante el proceso de molienda, los gránulos 
de almidón pueden ser lesionados, produciendo así lo que se denomina almidón dañado. La intensidad del daño depende principalmente de la fuerza con que se realiza la molienda y de la dureza del grano (Hoseney, 1994). El almidón dañado afecta las propiedades de las harinas, fundamentalmente modifica la capacidad de hidratación, ya que absorbe el $100 \%$ de su peso en agua, tres veces más que la que absorbe el almidón que se encuentra en los gránulos sanos (Bushuk, 1998). Este incremento en la capacidad de absorber agua tiene un efecto negativo en la elaboración de galletitas (Barrera et al., 2007).

En países como Estados Unidos e Inglaterra, los productos de panificación que no requieren un gran desarrollo de gluten, principalmente galletitas, son elaboradas generalmente con harinas provenientes de trigos blandos. En comparación con el trigo duro, el trigo blando ofrece una menor resistencia a la molienda y posee un menor contenido y calidad de proteínas. La harina de trigo blando presenta, menor tamaño medio de partícula, menor nivel de desarrollo de gluten y de almidón dañado y una menor capacidad de retención de agua (Doescher \& Hoseney, 1985; Rogers et al., 1993; Parey \& Delcour, 2008).

Una harina de trigo se considera que tiene buena calidad cuando produce galletitas dulces de gran diámetro con un patrón de agrietamiento uniforme de la superficie. Las galletitas de masa blanda, elaboradas a partir de masas que carecen de extensibilidad y elasticidad debido a la falta de una estructura de gluten desarrollada, y que son producidas con harinas de trigo blando, son muy diferentes a las fabricadas con harinas de trigo duro. Las galletitas obtenidas a partir de harina de trigo blando son de bajo espesor, gran diámetro y tiernas, mientras que las galletas hechas de harina de trigo duro son gruesas, duras y de menor diámetro (Miller \& Hoseney, 1997). Entre los parámetros que principalmente explican estas diferencias se encuentran el contenido y la calidad de las proteínas, el contenido de almidón dañado y de pentosanos y el tamaño de partículas (Manley, 2000, Torri et al., 2003).

Frente a la imposibilidad de contar con variedades más apropiadas para la elaboración de galletitas, la industria galletitera argentina utiliza harinas provenientes de trigo pan de baja calidad, a las que se le incorporan aditivos específicos para controlar la formación de gluten, o se producen modificaciones en los procesos de elaboración (Moiraghi et al., 2005). La calidad panificable de estas harinas se determina evaluando su absorción de agua, sus propiedades físicas y su comportamiento tecnológico durante el proceso de panificación
(Menkovska et al., 2002).

El objetivo del presente trabajo fue evaluar las propiedades físico-químicas de diferentes fracciones de harina de trigo pan obtenidas por molienda industrial y su calidad galletitera en comparación con una harina de trigo pan obtenida en un molino experimental.

\section{MATERIALES Y MÉTODOS}

\section{Muestras}

Se utilizaron cuatro fracciones de harinas de trigo pan obtenidas por molienda industrial y una harina de trigo pan obtenida por molienda experimental. La molienda industrial se llevó a cabo en el Molino Carlos Boero Romano SA (San Francisco, Córdoba, Argentina), cuya capacidad de molienda es de $210 \mathrm{t} /$ día, y la molienda experimental se realizó mediante un molino piloto Agromatic AG AQC 109 (Suiza) con capacidad de $2 \mathrm{~kg} / \mathrm{h}$. El trigo limpio fue cedido por Molino Carlos Boero Romano SA, acondicionado a $16 \%$ de humedad, almacenado en recipientes de polietileno cerrados durante 24 horas y molido posteriormente.

La Tabla 1 muestra los parámetros de producción de las fracciones de harina empleadas en este estudio. La muestra M1 se obtuvo por molienda en molino piloto Agromatic AG AQC 109 (Suiza), el cual posee un sistema de tres rodillos estriados. La harina se tamizó mediante un tamiz de $250 \mu$ de apertura. Las fracciones M2 y M5 se obtuvieron por el paso del material por tres etapas de roturas (R1: rotura con rodillos de dientes y estrías grandes, $\mathrm{R} 2$ y R3: reducción gradual del tamaño de dientes a presión de rotura semejante); las sémolas obtenidas pasaron por un sistema de dos grupos de cilindros de compresión (no estriados): M2, compresión fuerte (f) y tamización por tamiz plano de $118 \mu$ de apertura, y M5, compresión suave (s) y tamización por tamiz plano de $132 \mu$ de apertura. Las muestras M3 y M4 fueron sometidas al primer

Tabla 1. Parámetros de producción de las fracciones de harina utilizadas.

\begin{tabular}{cccc}
\hline $\begin{array}{c}\text { Fracción } \\
\text { de harina }\end{array}$ & $\begin{array}{c}\text { Número } \\
\text { de roturas }\end{array}$ & $\begin{array}{c}\text { Número de } \\
\text { compresión }\end{array}$ & $\begin{array}{c}\text { Tamiz plano }(\mu \\
\text { de abertura })\end{array}$ \\
\hline M2 & 3 & $2-f$ & 118 \\
M3 & 1 & $1-\mathrm{s}$ & 118 \\
M4 & 1 & $1-\mathrm{f}$ & 100 \\
M5 & 3 & $2-\mathrm{s}$ & 132 \\
M1 & Molino experimental & 250 \\
\hline
\end{tabular}

f: compresión fuerte, s: compresión suave 
paso de rotura (R1: rotura con rodillos de dientes y estrías grandes), y las sémolas separadas se comprimieron por un paso en cilindros de compresión (planos): M3, compresión suave y tamización por tamiz plano de $118 \mu$ de apertura, y M4, compresión fuerte y tamización por tamiz plano de $100 \mu$ de apertura.

\section{Análisis químicos y físico-químicos de las ha- rinas}

La composición y calidad de las harinas se determinó a través de ensayos fisicoquímicos como: contenido de humedad (Norma 15850, IRAM, 1990), contenido de cenizas (Norma 15851, IRAM, 2009), contenido de gluten (Norma 15864, IRAM, 2007), índice de caída (Norma 15862, IRAM, 2003), índice de sedimentación en dodecil sulfato de sodio (IS-SDS) (Dick \& Quick, 1983), perfil de capacidad de retención de solventes (SRC) (Método 56-11, AACC, 2000). El contenido de almidón dañado fue determinado mediante hidrólisis enzimática con $\alpha$-amilasa fúngica (Método 76-30 A, AACC, 1995) y posterior titulación de azúcares reductores (Método 80-60, AACC, 1995).

\section{Estudios reológicos}

La resistencia de las masas a una deformación de extensión multiaxial se evaluó mediante un alveógrafo de Chopin. Para este ensayo se preparó una masa con $250 \mathrm{~g}$ de harina, agua y cloruro de sodio (según el porcentaje de humedad de la harina), con la que se obtuvieron cinco piezas en forma de disco. A continuación, a partir del aire insuflado durante el ensayo se formó una burbuja con cada una de las piezas, y se registró la presión en su interior. La curva graficada está relacionada con la resistencia de la masa a la deformación (Método 54-30, AACC, 1995)

\section{Elaboración de galletitas}

Se elaboraron galletitas con las harinas en estudio. Los ingredientes utilizados fueron: harina 45 g, azúcar impalpable $27 \mathrm{~g}$, grasa vegetal 20,20 g, leche en polvo $2,25 \mathrm{~g}$, bicarbonato de sodio 0,50 $\mathrm{g}$, sal $0,42 \mathrm{~g}$ y agua $8,5 \mathrm{~mL}$. Las galletitas fueron horneadas a $180^{\circ} \mathrm{C}$ por 10 min (León et al., 1996).

\section{Evaluación de la calidad de las galletitas}

La calidad se determinó mediante el factor galletita (FG), obtenido de la relación entre el diáme- tro y la altura de cuatro galletitas orientadas al azar (León et al., 1996).

En todos los casos se examinó el color de la superficie de las galletitas, firmeza y estructura e irregularidad superficial de éstas. La determinación del color superficial se realizó a las 24 h de horneadas, con un espectrofotómetro de reflectancia (Minolta CM-500 d series). Se utilizó un ángulo del observador de $10^{\circ}$, iluminante D65, componente especular incluido, utilizando la escala CIELab ( $L^{*}$ : luminosidad, $a^{*}$ : rojo-verde y $b^{*}$ : amarillo - azul). Las determinaciones se realizaron sobre seis galletitas.

La firmeza de las galletitas se evaluó a las 24 h de horneadas, con un texturómetro INSTRON (Universal Testing Machine, modelo 3342, EUA). Se realizó una prueba de quiebre de tres puntos. El producto fue apoyado sobre dos soportes paralelos, separados por una distancia de $4,5 \mathrm{~cm}$. Un tercer eje paralelo, del mismo material de los soportes fue desplazado verticalmente ejerciendo una fuerza de compresión hasta producir un quiebre en la estructura del producto. La velocidad de desplazamiento del equipo fue de $0,5 \mathrm{~mm} / \mathrm{s}$. Los resultados de las experiencias instrumentales se expresaron mediante un gráfico fuerza vs deformación. Los valores de fuerza máxima se relacionaron con la dureza de las galletitas (Gaines, 1994). Las determinaciones se realizaron sobre cuatro galletitas por lote.

La estructura superficial de las galletas se evaluó a través de análisis de imagen. Las galletitas fueron escaneadas con un escáner fotográfico (HP Scanjet G3010, EUA). Las imágenes RGB en formato de archivo TIFF se analizaron con el programa de análisis de imágenes Image J v1,410 (National Institutes of Health, EUA). Se seleccionó un área circular $(\mathrm{ROI})$ representativa (850 píxeles de diámetro) de la parte central de cada galletita en todos los lotes. Las ROIs se convirtieron a 8-bits (0 negro, 255 blanco). La imagen seleccionada fue segmentada por un valor de gris para crear una imagen binaria mediante el algoritmo Renyi Entropy (Kapur et al., 1985). De este modo, todos los píxeles con nivel de gris por encima del umbral se mostraron en blanco y fueron considerados superficie no agrietada; a su vez, los pixeles con nivel de gris por debajo del umbral se mostraron en negro y fueron considerados superficie agrietada. El análisis de las imágenes permitió obtener la fracción de área (FA) que corresponde a grieta en la superficie de la galletita.

La irregularidad superficial de las galletas fue caracterizada mediante la dimensión fractal de la textura superficial (D), mediante el software Ima- 
ge $J$ utilizando el algoritmo de "conteo de cajas" (Fractal Box count). Este parámetro es una medida de la complejidad de la textura y es relevante cuando las dimensiones euclideanas tales como diámetro y longitud no son buenos descriptores de la complejidad. El comando cuenta el número de cajas necesarias para cubrir el contorno de un objeto binario de un pixel (Smith et al., 1996). El algoritmo genera un gráfico con el log del tamaño en el eje $X$ y el log del recuento en el eje Y. La pendiente de la línea es el negativo de la dimensión fractal (D). Los tamaños de caja utilizados fueron de 2, 3, 4, 6, 8, 12, 16, 32 y 64 pixeles (plugin Fractal Count del programa ImageJ).

Al menos cuatro galletitas por lote fueron analizadas.

\section{Análisis estadístico}

En todos los casos las determinaciones se realizaron por duplicado y los resultados se informaron como el valor promedio. Los datos obtenidos se trataron estadísticamente mediante un análisis de varianza, y los resultados fueron comparados por el método de la mínima diferencia significativa (LSD) de Fisher a un nivel de significación del 0,05.

Los análisis de correlación simple entre variables fueron evaluados por el método de Pearson. El coeficiente de correlación de Pearson es una medida de la magnitud de la asociación lineal entre dos variables que no depende de las unidades de medida de las variables originales.

Las relaciones entre las características de las galletitas y los parámetros físico-químicos y reológicos de las fracciones de harinas fueron estudiadas por análisis de componentes principales (ACP). El ACP construye ejes artificiales (componentes principales) que permiten obtener gráficos de dispersión de observaciones y variables para la interpretación de la variabilidad de las observaciones. Mediante este análisis es posible visualizar las observaciones y variables en un mismo espacio, lo cual permite identificar asociaciones entre observaciones, entre variables y entre variables y observaciones.

El análisis estadístico se realizó mediante el programa INFOSTAT (Di Rienzo et al., 2011).

\section{RESULTADOS Y DISCUSIÓN}

Los procedimientos de molienda realizados para la obtención de las muestras no modificaron significativamente el contenido de proteína de las harinas. Las fracciones de harinas estudiadas presentaron un rango de porcentaje de proteínas que varió entre 10,75 y 11,78 g/100 g harina (Tabla 2). Sin embargo, el contenido de gluten cambió significativamente entre 22,3 y $27,5 \%$; la harina obtenida en el molino experimental y M5 tuvieron el mayor y menor valor respectivamente.

En las muestras de harina analizadas se registraron variaciones significativas en el contenido de cenizas y almidón dañado. El contenido de cenizas presentó valores entre 0,51 y $0,77 \mathrm{~g} / 100 \mathrm{~g}$ harina. El mayor contenido de cenizas fue el registrado en las fracciones M2 y M5, obtenidas a partir de etapas sucesivas de roturas y de compresión. Estos resultados se explican por la contaminación con salvado que se origina durante estos procedimientos. Así mismo, la fracción M1 también mostró un alto grado de contaminación con salvado, probablemente por la apertura de tamiz utilizada $(250 \mu)$.

El contenido de almidón dañado presentó valores entre 8,49 y $12,79 \%$. Las muestras M3 y M4 exhibieron los valores de almidón dañado más altos, lo que indica que el proceso de molienda del endospermo separado en los primeros rodillos de rotura, a través de un sólo paso por rodillos de compresión, involucró una gran energía y lesionó en gran medida los gránulos obtenidos. M4, que fue obtenida por una etapa de compresión más fuerte que M3, presentó el mayor contenido de al-

Tabla 2. Composición de las harinas estudiadas.

\begin{tabular}{cccccccc}
\hline Muestra & Humedad (\%) & \multicolumn{2}{c}{ Proteínas (\%) } & Cenizas (\%) & Gluten (\%) & AD (\%) \\
\hline M1 & $12,76 \pm 0,07 \mathrm{a}$ & $11,77 \pm 0,01 \mathrm{~b}$ & $0,77 \pm 0,02 \mathrm{c}$ & $27,5 \pm 0,6 \mathrm{~b}$ & 8,49 & $\pm 0,21 \mathrm{a}$ \\
M2 & $13,48 \pm 0,02 \mathrm{~b}$ & $11,76 \pm 0,33 \mathrm{~b}$ & $0,66 \pm 0,02 \mathrm{~b}$ & $26,1 \pm 0,5 \mathrm{~b}$ & 9,31 & $\pm 0,21 \mathrm{a}$ \\
M3 & $14,19 \pm 0,33 \mathrm{c}$ & $11,78 \pm 0,09 \mathrm{~b}$ & $0,51 \pm 0,02 \mathrm{a}$ & $25,9 \pm 0,5 \mathrm{~b}$ & $10,97 \pm 0,31 \mathrm{~b}$ \\
M4 & $14,05 \pm 0,26 \mathrm{bc}$ & $10,75 \pm 0,18 \mathrm{a}$ & $0,51 \pm 0,02 \mathrm{a}$ & $23,7 \pm 0,5 \mathrm{a}$ & $12,79 \pm 0,41 \mathrm{c}$ \\
M5 & $14,28 \pm 0,00 \mathrm{c}$ & $11,59 \pm 0,02 \mathrm{~b}$ & $0,77 \pm 0,02 \mathrm{c}$ & $22,3 \pm 0,4 \mathrm{a}$ & $10,39 \pm 0,27 \mathrm{~b}$ \\
\hline
\end{tabular}

Letras diferentes indican diferencias significativas $(p \leq 0,05)$. 
midón dañado.

El perfil de capacidad de retención de solventes (SRC), inicialmente propuesto para evaluar la calidad de trigos blandos (Slade \& Levine, 1994; Gaines, 2000), se ha aplicado con éxito en trigos pan (Moiraghi et al., 2005; Colombo et al., 2008). Es una prueba de solvatación para harinas basada en la capacidad de retención de agua (SRC-agua), sacarosa $50 \%$ (SRC-sac), carbonato de sodio $5 \%$ (SRC-car) y ácido láctico 5\% (SRC-lac) de las redes poliméricas, después de mezclar y centrifugar las suspensiones. Cada solvente puede predecir la contribución funcional de los componentes de la harina: el SRC-agua es afectado por todos los constituyentes hidrofílicos de la harina y se relaciona con la capacidad de retención de agua por los componentes poliméricos, el SRC-lac está asociado con la capacidad de formación de redes de la fracción de gluteninas y la fuerza del gluten, el SRC-car se vincula con el contenido de almidón dañado, y el SRC-sac está relacionado con el contenido de pentosanos accesibles a la fase líquida (Gaines, 2000; Guttieri et al., 2002; Kweon et al., 2009).

El índice de sedimentación en SDS (IS-SDS), utilizado para determinar la calidad panadera de las muestras de harina, evalúa la capacidad del gluten de formar una red y se relaciona con el volumen específico de las piezas del pan (Dick \& Quick, 1983).

De los resultados obtenidos se observó que el SRC-agua presentó valores entre 65,7 y $71,9 \%$, M1 presentó el valor mínimo y M4 y M5 los valores más altos. El SRC-sac mostró un rango de valores entre 99,0 y 113,8\%, M1 presentó el menor valor y M3 el mayor. El SRC-car y el SRC-lac variaron entre 80,5 y $90,7 \%$ y 107,8 y $127,8 \%$, respectivamente. En estos casos M1 presentó el menor valor y M4 el mayor. Los resultados se encuentran en el mismo rango que los informados por Moiraghi et al. (2005) y Colombo et al. (2008) para diferentes variedades de trigo pan.

Los valores del IS-SDS cambiaron entre 10,25 y $16,50 \mathrm{~cm}^{3}$. La muestra M1 presentó el menor valor y M4, el mayor. Estos valores fueron algo más altos a los obtenidos por Moiraghi et al. (2005), pero algo más bajos que los publicados por Colombo et al. (2008) para diferentes variedades de trigo pan.

Los valores de los índices SRC y IS-SDS mostraron diferencias significativas para las distintas fracciones de harina (Tabla 3), lo que indica que las variaciones en la composición, generadas por el proceso de molienda, modificaron la habilidad de las harinas para retener los diferentes solventes y, probablemente, el comportamiento en el proceso de panificación.

El comportamiento de las masas de harina de trigo puede ser caracterizado por parámetros reológicos, tales como la elasticidad, la extensibilidad y la tenacidad. Estas propiedades de las masas de trigo son de particular importancia en los procesos de elaboración de los distintos productos horneados. El análisis alveográfico de las fracciones no mostró diferencias significativas $(p \leq 0,05)$ entre las fracciones de las harinas en estudio (Tabla 4). La

Tabla 3. Efecto de la molienda sobre el índice de sedimentación en SDS (SDS-IS) y el perfil de capacidad de retención de solventes (SRC) de las harinas.

\begin{tabular}{|c|c|c|c|c|c|c|c|}
\hline Muestra & IS-SDS $\mathrm{cm}^{3}$ & & SRC-Agua ( & & SRC-Sac (\%) & SRC-Car (\%) & SRC-Lac (\%) \\
\hline M1 & $10,25 \pm 0,25$ & a & $65,7 \pm 0,7$ & $\mathrm{a}$ & $99,0 \pm 1,1 \mathrm{a}$ & $80,5 \pm 0,3 a$ & $107,8 \pm 1,8 a$ \\
\hline M2 & $14,75 \pm 0,25$ & $\mathrm{~b}$ & $69,4 \pm 0,3$ & $b$ & $105,0 \pm 0,5 a b$ & $86,2 \pm 0,7 b$ & $110,0 \pm 0,1 \quad a$ \\
\hline M3 & $15,50 \pm 0,00$ & C & $69,3 \pm 0,9$ & $b$ & $111,7 \pm 3,9 \mathrm{bc}$ & $87,1 \pm 0,7 \mathrm{bc}$ & $126,6 \pm 0,3 b$ \\
\hline M4 & $16,50 \pm 0,00$ & $d$ & $71,9 \pm 0,0$ & c & $113,8 \pm 1,2 \mathrm{c}$ & $90,7 \pm 1,9 \mathrm{c}$ & $127,8 \pm 0,5 b$ \\
\hline M5 & $16,25 \pm 0,25$ & d & $71,9 \pm 0,7$ & C & $107,9 \pm 2,7 \mathrm{bc}$ & $89,0 \pm 0,5 b c$ & $108,6 \pm 1,8 a$ \\
\hline
\end{tabular}

Letras diferentes indican diferencias significativas $(p \leq 0,05)$.

Tabla 4. Efecto de la molienda sobre parámetros reológicos de las fracciones de harina. Índice de caída (FN) y parámetros alveográficos.

\begin{tabular}{|c|c|c|c|c|c|c|c|c|c|c|c|c|c|c|c|}
\hline Muestra & \multicolumn{3}{|c|}{$\mathrm{FN}(\mathrm{s})$} & \multicolumn{3}{|c|}{ W (10-4 J) } & \multicolumn{3}{|c|}{$\mathrm{P}(\mathrm{mm})$} & \multicolumn{3}{|c|}{$\mathrm{L}(\mathrm{mm})$} & \multicolumn{3}{|c|}{$P / L$} \\
\hline M1 & 483 & \pm 36 & $a$ & 256 & \pm 41 & $a$ & 88 & \pm 15 & $a$ & 90,0 & \pm 14 & $a$ & 1,0 & \pm 0 & $2 \mathrm{a}$ \\
\hline M2 & 501 & \pm 38 & a & 382 & \pm 61 & a & 116 & \pm 19 & a & 82,0 & \pm 12 & a & 1,4 & \pm 0 & , $2 \mathrm{a}$ \\
\hline M3 & 446 & \pm 33 & $a$ & 353 & \pm 56 & $a$ & 125 & \pm 21 & $a$ & 65,0 & \pm 10 & a & 1,9 & \pm 0 & , $3 \mathrm{a}$ \\
\hline M4 & 457 & \pm 34 & $a$ & 309 & \pm 49 & $a$ & 114 & \pm 19 & $a$ & 64,0 & \pm 10 & a & 1,8 & \pm 0 &, $3 a$ \\
\hline M5 & 455 & \pm 34 & a & 276 & \pm 44 & a & 118 & \pm 20 & a & 57,0 & \pm 9 & a & 2,1 & \pm 0 &, $3 a$ \\
\hline
\end{tabular}

Letras diferentes indican diferencias significativas $(p \leq 0,05)$. 
actividad $\alpha$-amilásica, representada indirectamente por el número de caída, no mostró diferencias significativas entre las fracciones estudiadas. El análisis alveográfico y el número de caída son metodologías muy utilizadas en la caracterización de harinas en el ámbito industrial (León \& Rosell 2007); sin embargo, en este estudio no sirvieron para diferenciar entre las distintas fracciones de harina obtenidas.

Las galletitas elaboradas con las fracciones de harina estudiadas se muestran en la Figura 1. La Tabla 5 y 6 muestran las características de las galletitas elaboradas con las harinas de trigo pan. El factor galletita (FG) presentó valores entre 4,8 y 6,1. Las muestras M1 y M4 tuvieron el mayor y menor valor de FG, respectivamente. Los valores de FG mostraron correlación inversa $(p \leq 0,05)$ con los valores de IS-SDS $(r=-0,98)$, de SRC-agua $(r=$ $-0,89)$, de SRC-sac $(r=-0,79)$, SRC-carb $(r=-0,94)$ y el contenido de almidón dañado $(r=-0,86)$. Estos resultados fueron similares a los obtenidos por Colombo et al. (2008) y corroboran el efecto negativo de los componentes hidrofílicos en la calidad de las galletas. Los parámetros de color superficial de las galletitas no cambiaron significativamente con las fracciones de harinas evaluadas (Tabla 5).

Generalmente, la masa de galletitas dulces no posee propiedades elásticas, es decir que cede a una fuerza de tracción, y es de consistencia variable. La harina es el ingrediente principal de las masas para galletitas, pero son necesarias grandes cantidades de grasa y de azúcar, no sólo por el aporte del sabor, aroma y color del producto final, sino para generar plasticidad y cohesividad

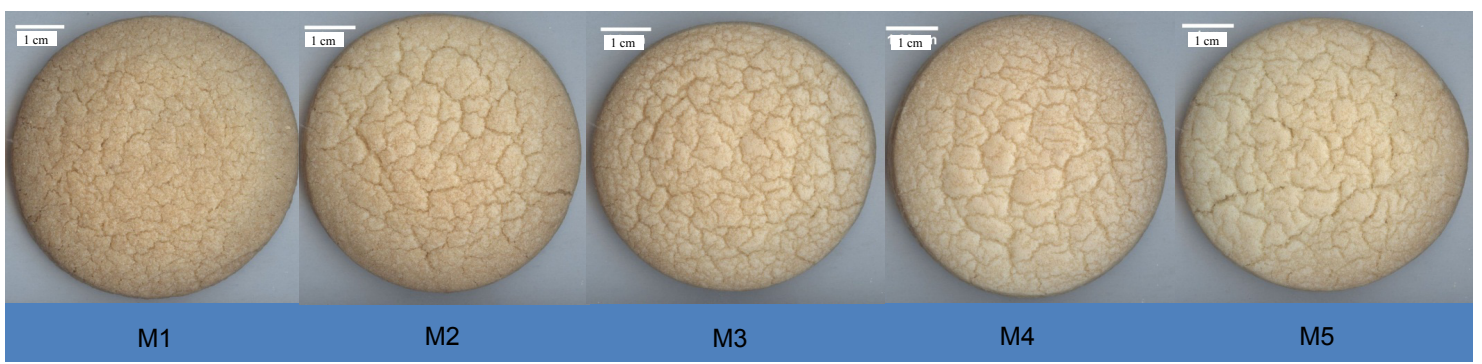

Figura 1. Galletitas elaboradas con las fracciones de harinas analizadas.

Tabla 5. Parámetros de calidad de las galletitas. Factor galletita y color.

\begin{tabular}{|c|c|c|c|c|c|}
\hline Muestra & $F G$ & & $L^{*}$ & $a^{*}$ & $b^{*}$ \\
\hline M1 & $6,1 \pm 0,1$ & $d$ & $65,4 \pm 0,4 a$ & $9,4 \pm 0,3$ & $32,2 \pm 0,2 a$ \\
\hline M2 & $5,2 \pm 0,1$ & c & $67,2 \pm 0,4 a$ & $10,6 \pm 0,3$ & $33,0 \pm 0,5 a$ \\
\hline M3 & $4,8 \pm 0,1$ & $b$ & $69,7 \pm 0,3 a$ & $9,4 \pm 0,1$ & $30,9 \pm 0,3 a$ \\
\hline M4 & $4,5 \pm 0,0$ & a & $71,3 \pm 0,1 \quad a$ & $8,9 \pm 0,0$ & $29,8 \pm 0,2 a$ \\
\hline M5 & $4,7 \pm 0,1$ & $a b$ & $71,6 \pm 2,6 a$ & $7,9 \pm 1,6$ & $30,5 \pm 1,3 a$ \\
\hline
\end{tabular}

Letras diferentes indican diferencias significativas $(p \leq 0,05)$.

Tabla 6. Parámetros de calidad de las galletitas. Dureza (fuerza máxima), fracción de área (FA) y dimensión fractal de la textura superficial (D).

\begin{tabular}{|c|c|c|c|c|c|c|c|c|c|c|c|c|}
\hline \multirow{2}{*}{$\begin{array}{c}\text { Muestra } \\
\text { M1 }\end{array}$} & \multicolumn{4}{|c|}{$F(k g f)$} & \multicolumn{4}{|c|}{$\mathrm{FA}$} & \multicolumn{4}{|c|}{$D$} \\
\hline & 6,5 & \pm & 0,1 & $a$ & 7,9 & \pm & 1,2 & $\mathrm{a}$ & 1,38 & \pm & 0,04 & $a b$ \\
\hline M2 & 7,0 & \pm & 0,0 & $\mathrm{a}$ & 8,8 & \pm & 1,8 & $\mathrm{a}$ & 1,41 & \pm & 0,05 & bc \\
\hline M3 & 11,4 & \pm & 1,9 & $b$ & 11,5 & \pm & 1,4 & $b$ & 1,46 & \pm & 0,04 & c \\
\hline M4 & 11,9 & \pm & 0,9 & $b$ & 11,6 & \pm & 2,1 & $b$ & 1,47 & \pm & 0,05 & $c$ \\
\hline M5 & 7,6 & \pm & 0,4 & $a$ & 6,8 & \pm & 1,8 & $a$ & 1,33 & \pm & 0,06 & $\mathrm{a}$ \\
\hline
\end{tabular}

Letras diferentes indican diferencias significativas $(p \leq 0,05)$. 
de la masa con mínima o ninguna formación de la red de gluten. La estructura de este tipo de galletitas consiste en una mezcla de proteína, almidón y azúcar (azúcar fundido sobre enfriado), la cual no presenta una matriz proteica continua y la grasa se encuentra en forma de grandes glóbulos interconectados entre cuerpos de almidón y de proteínas. La textura es gruesa por la gran coalescencia de las burbujas de gas que se forman durante la cocción (Manley, 2000).

El ensayo de quiebre de tres puntos es una prueba de flexión y consiste en evaluar la fuerza máxima necesaria para producir un quiebre total de la estructura del producto (Gaines, 1994). La fuerza máxima (dureza) de ruptura de las galletitas mostró cambios significativos según la fracción de harina utilizada; se encontraron dos grupos, el de mayor dureza integrado por M3 y M4 y el de menor dureza formado por M1, M2 y M5 (Tabla 6). La dureza mostró correlación $(p \leq 0,05)$ inversa con el FG $(r=-0,68)$, lo que puede estar relacionado con un mayor espesor de las galletitas.

Las galletitas dulces tienen como requisito de calidad, además de piezas de gran diámetro, un alto grado de agrietamiento y un patrón de agrietamiento uniforme de la superficie. En este trabajo se evaluaron las características superficiales de las galletitas mediante la fracción de área (FA), que corresponde a la fracción de grieta en la superficie de la galletita, y la dimensión fractal de la textura superficial (D), que provee una descripción numérica de la morfología de los objetos que poseen estructuras complejas e irregulares. El parámetro $D$ es especialmente relevante en los casos en que las medidas euclídeas tales como el diámetro o longitud no son buenos descriptores de complejidad. Este último parámetro se ha utilizado para explicar los cambios en la estructura de productos panificados como consecuencia de su procesamiento (Quevedo et al., 2002, Pérez-Nieto et al., 2010).

La fracción de área de agrietamiento de la superficie de las galletitas mostró cambios significativos según la fracción de harina utilizada; así se encontraron dos grupos, el de mayor FA, integrado por M3 y M4, y el de menor FA, formado por M1, M2 y M5 (Tabla 6). Este parámetro mostró la misma tendencia que la fuerza de quiebre de las galletitas. La FA de agrietamiento mostró correlación directa $(p \leq 0,05)$ con el contenido de DS $(r=0,70)$, con el SRC-lac $(r=0,88)$ y con la firmeza $(r=0,78)$ e inversa con el contenido de cenizas $(-0,89)$.

La dimensión fractal varió significativamente entre 1,33 y 1,47, para M5 y M4, respectivamente. Un mayor valor de $\mathrm{D}$ se relaciona con superficies más complejas o rugosas (Quevedo et al., 2002), lo que sugiere que M4 y M5 produjeron galletitas

Figura 2. Análisis de componentes principales. Relación entre las características de las galletitas y los parámetros físico-químicos y reológicos de las fracciones de harinas.

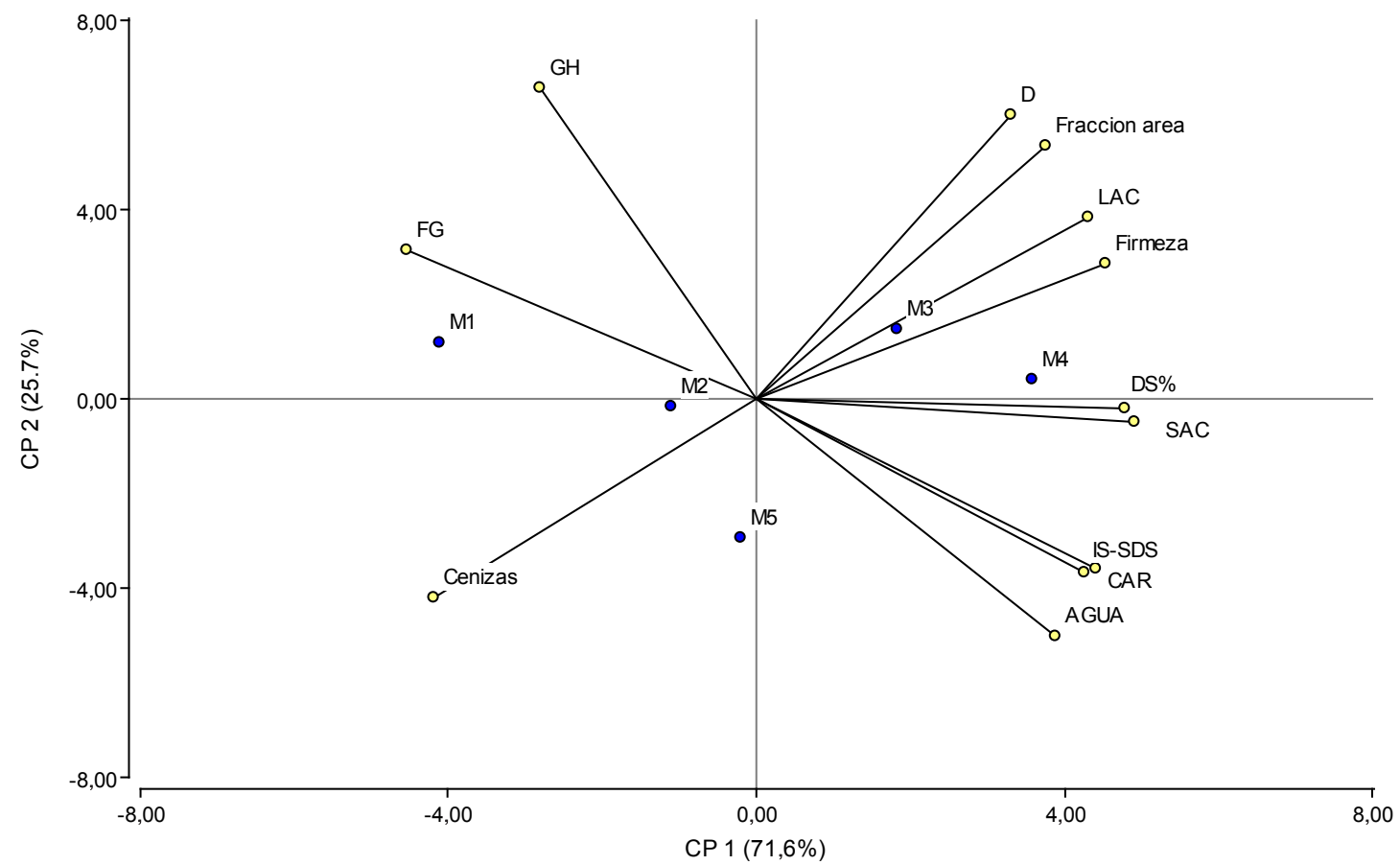


con mayor rugosidad superficial y $\mathrm{M} 5$ el menor valor. El parámetro $D$ correlacionó directamente $(p \leq 0,05)$ con el SRC-lac $(r=0,83)$ y firmeza $(0,73)$ e inversamente con el contenido de cenizas $(-0,87)$. Las propiedades superficiales, FA y D, parecen estar relacionadas con la calidad proteica, ya que el SRC-lac se asocia con las características de las gluteninas y la fuerza del gluten.

Los parámetros que mostraron diferencias significativas y coeficientes de correlación significativos fueron analizados mediante el análisis de componentes principales (ACP), lo que permitió evaluar la variabilidad entre las muestras en función de los parámetros determinados.

Para representar el mayor porcentaje de variabilidad de las muestras de harina se utilizaron dos componentes, lo que permitió explicar el 97,3\% de la variabilidad total. Los componentes principales 1 (CP1) y 2 (CP2) describieron el 71,6 y el $25,7 \%$ de la variación respectivamente (Fig 2). El 71,6\% de la variabilidad de las muestras fue explicado por IS-SDS, SRC, \%DS, cenizas, FG, dureza de las galletitas y propiedades superficiales, ya que estas fueron las variables de mayor proyección, es decir de mayor peso, sobre el eje CP1. Los pesos de las variables fueron similares, por lo que el análisis sugiere contribuciones similares de cada variable sobre la variabilidad de las muestras. Por otro lado, el $25,7 \%$ de la variabilidad de éstas fue explicado por SRC-agua, gluten húmedo y las propiedades superficiales de las galletitas, debido a que estas variables fueron las de mayor peso sobre el eje CP2.

La componente principal 1 fue definida por ISSDS, SRC, almidón dañado, dureza de las galletitas y propiedades superficiales a lo largo del eje positivo. En esta región se ubicaron las muestras M3 y M4, ambas muestras producidas por compresión del endospermo separado en la primer etapa de rotura. La región negativa del eje CP1 fue descripta por el gluten húmedo, cenizas y el factor galletita, e incluyó a las muestras M1, M2 y M5.

La componente principal 2 fue principalmente definida por SRC-lac, propiedades superficiales, dureza de las galletitas, factor galletita y gluten húmedo a lo largo del eje positivo. En esta región se ubicaron las muestras M1, M3 y M4. Por otro lado, en eje CP2 negativo se encontró el contenido de cenizas, almidón dañado, SRC-agua, SRC-carb, SRC-sac, IS-SDS. En esta región del gráfico sólo se ubicaron las muestras M2 y M5, las cuales fueron producidas por compresión del endospermo separado luego de tres etapas de rotura.

Los resultados muestran que el análisis de componentes principales permitió discriminar entre las diferentes fracciones de harinas, y que M3 y M4 fueron similares en términos de almidón dañado, dureza y propiedades superficiales de las galletitas, IS-SDS y SRC. Las muestras M2 y M5 fueron similares en relación al contenido de cenizas, almidón dañado, SRC-agua, SRC-carb, SRC-sac, IS-SDS. Además, los parámetros gluten húmedo, cenizas y factor galletita permitieron diferenciar la muestra M1, la única fracción obtenida en molino piloto y con mayor tamaño de partícula, de las demás fracciones.

\section{CONCLUSIONES}

Los resultados mostraron que la evaluación físico-química de las harinas combinada con la evaluación reológica pudieron predecir el comportamiento tecnológico de una harina en la elaboración de galletitas.

El proceso de molienda afectó significativamente la composición de las harinas. Las fracciones de harina presentaron diferencias significativas con respecto a los parámetros de calidad de las galletitas, factor galletita y aspecto superficial. Las propiedades reológicas proporcionaron menor información que las características físico-químicas de las harinas. El contenido de almidón dañado y el resto de los componentes hidrofílicos de las harinas mostraron una gran influencia sobre los parámetros de calidad de las harinas.

En general, los resultados obtenidos mostraron que las harinas analizadas no presentaron un buen desempeño en la elaboración de galletitas. Sin embargo, de las cuatro fracciones de harina de trigo pan obtenidas por molienda industrial, la fracción de harina M2 mostró el mayor factor galletita, el menor valor de firmeza y los valores más bajos de absorción, lo que indica que es la fracción de harina que mejores parámetros de calidad presenta para la elaboración de galletitas. El comportamiento presentado por la fracción de harina M2 se asoció principalmente a menores valores de almidón dañado y de absorción de solución de sacarosa, confirmando el efecto negativo de los componentes hidrofílicos de las harinas sobre la calidad de las galletitas.

\section{AGRADECIMIENTOS}

El presente trabajo fue financiado por la Secretaría de Ciencia y Tecnología de la Universidad Nacional de Córdoba y el Ministerio de Ciencia y Tecnología de Córdoba. 


\section{BIBLIOGRAFÍA}

AACC (American Association of Cereal Chemists), 2000. Approved Methods of the AACC, 9th edn., The Association, USA.

Barrera, G.N.; G.T. Perez, P.D. Ribotta and A.E. León, 2007. Influence of damaged starch on cookie and bread-making quality. European Food Research and Technology 225:1-7.

Bushuk, W., 1998. Interactions in wheat doughs. In: Hamer \& Hoseney (eds.) Interactions the key to cereal quality. American Association of cereal chemists. Inc. St Paul MN, USA. pp 1-16.

Colombo, A.; G.T. Perez, P.D. Ribotta and A.E. León, 2008. A comparative study of physicochemical tests for quality prediction of Argentine wheat flours used as corrector flours and for cookie production. Journal of Cereal Science 48:775-780.

Dewettinck, K.; F. Van Bockstaele, B. Kuhne, D. Van de Walle, T.M. Courtens and X. Gellynck, 2008. Nutritional value of bread: Influence of processing, food interaction and consumer perception. Journal of Cereal Science 48:243-257.

Dick, J. and J. Quick, 1983. A modified screening test for rapid estimation of gluten strength in early-generation durum wheat breeding lines. Cereal Chemistry 60:315-318.

Doescher, L. and R.C. Hoseney, 1985. Effect of sugar type and flour moisture on surface cracking of sugarsnap cookies. Cereal Chemistry 62:263-266.

Di Rienzo J.A.; F. Casanoves, M.G. Balzarini, L. Gonzalez, M. Tablada, C.W. Robledo. InfoStat versión 2011. Grupo InfoStat, FCA, Universidad Nacional de Córdoba, Argentina. URL http://www.infostat.com.ar.

Gaines C.S., 1994. The Science of Cookie and Cracker Production. Faried H. (eds). New York. pp. 455 - 495.

Gaines, C.S., 2000. Collaborative study of methods for solvent retention capacity profiles (AACC Method 5611). Cereal Foods World 45:303-306.

Guttieri, M.J.; R. McLean, S.P. Lanning, L.E. Talbert and E. Souza, 2002. Assessing environmental influences on solvent retention capacities of two soft white spring wheat cultivars. Cereal Chemistry, 79:880-884.

Hoseney, R.C., 1994. Principles of Cereal Science and Technology, 2nd edn., American Association of Cereal Chemists, Inc., St. Paul, MN, USA.

IRAM (Instituto argentino de normalización y certificación de materiales). Norma IRAM 15850, 1990. Método práctico para la determinación de humedad en granos, harinas y subproductos. Cereales y productos de cereales. Determinación del contenido de humedad. Buenos Aires, Argentina.

IRAM (Instituto argentino de normalización y certificación de materiales). Norma IRAM 15851, 2009. Cereales y productos de cereales. Determinación de cenizas por incineración. Buenos Aires, Argentina.

IRAM (Instituto argentino de normalización y certificación de materiales). Norma IRAM 15862, 2003. Cereales. Determinación de la actividad de la alfa-amilasa (número de caída), según Hagberg-Perten. Buenos Aires, Argentina.

IRAM (Instituto argentino de normalización y certificación de materiales). Norma IRAM 15864, 2007. Harina de trigo, Índice de gluten. Método de lavado automático. Buenos Aires, Argentina.

Kapur, J.N.; P.K. Sahoo and A.C.K. Wong, 1985. A new method for gray-level picture thresholding using the entropy of the histogram. Graph. Models Image Process. 29:273-285.

Kweon, M.; L. Slade, H. Levine, R.J. Martin, L.C. Andrews and E.J. Souza, 2009. Effects of Extent of Chlorination, Extraction Rate, and Particle Size Reduction on Flour and Gluten Functionality Explored by Solvent Retention Capacity (SRC) and Mixograph. Cereal Chemistry 86:221-224.

León, A.E.; O.J. Rubiolo and M.C. Añón, 1996. Use of triticale flours in cookies: quality factors. Cereal Chemistry 73:779-784.

León, A.E y Rosell, C.M, 2007. De tales harinas, tales panes: Granos, harinas y productos de panificacion en Iberoamérica. Capitulo 1: Trigo. 1a edición, Cyted y Báez Ediciones. Córdoba, Argentina. ISBN 9789871311071.

Manley, D.J.R., 2000. Short dough biscuits, in Technology of Biscuits, Crackers, and Cookies, Third Edition. Woodhead Publishing, Cambridge. pp. 274-282.

Miller, R.A. and R.C. Hoseney, 1997. Factors in Hard Wheat Flour Responsible for Reduced Cookie Spread. Cereal Chemistry 74:330-336.

Menkovska, M.; D. Knezevic and M. Ivanoski, 2002. Protein allelic composition, dough rheology, and baking characteristics of flour mill streams from wheat cultivars with known and varied baking qualities, Cereal Chemistry 79:720-725.

Moiraghi, M.; P.D. Ribotta, A. Aguirre, G.T. Pérez y A.E. León, 2005. Análisis de la aptitud de trigos pan para la elaboración de galletitas y bizcochuelos. Agriscientia XXII (2):47-54

Parey, B. and J.A. Delcour, 2008. The role of wheat flour constituents, sugar, and fat low moisture cereal based products: A review on sugarsnap cookies. Food Science Nutrition 48:824-839.

Pérez-Nieto, A.; J.J. Chanona-Pérez, R.R. Farrera-ReboIlo, G.F. Gutiérrez-López, L. Alamilla-Beltrán and G. Calderón-Domínguez, 2010. Image analysis of structural changes in dough during baking. LWT - Food 
Science and Technology, 43:535-543.

Prabhasankar, P.; M.L. Sudha and P. Haridas Rao, 2000. Quality characteristics of wheat flour milled streams. Food Research. Int. 33:381-386.

Quevedo, R.; C.G. Lopez, J.M. Aguilera and L. Cadoche, 2002. Description of food surfaces and microstructural changes using fractal image texture analysis. Journal of Food Engineering 53:361-371.

Rogers, D.E.; R.C. Hoseney, G.L. Lookhart, S.P. Curran, W.D.A. Lin and R.G. Sears, 1993. Milling and cookie baking quality of near isogenic lines of wheat differing in kernel hardness. Cereal Chemistry 70:183-187.

Ruffet, M. J, 1994. Break roller mill performances. In: Primary cereal processing. Godon, B. and Willm, C. eds. VCH Publishing: New York. Pp 189-210.
Slade, L. and H. Levine, 1994. Structure-function relationships of cookie and cracker ingredients. In: The science of cookie and cracker production. Faried H. (ed.). Chapman \& Hall/A VI, New York. pp. 23-141.

Smith, T.G.; G.D. Lange and W.B. Marks, 1996. Fractal Methods and Results in Cellular Morphology, Journal Neuroscience Methods, 69:1123-126.

Torri, C.L.; P.D. Ribotta, M.H. Morcillo, O.J. Rubiolo, G.T. Pérez y A.E. León, 2003. Determinación del contenido de almidón dañado en harinas de triticale. Su influencia sobre la calidad galletitera. Agriscientia, 20:3-8.

Webb, C. and G.W. Owens, 2003. Milling and flour quality. In Bread making Improving quality. Stanley P. Cauvain (eds) CRC Press LLC, EUA. Capitulo 3. 